\title{
La estrategia Europa 2020 y la Sociedad de la Información como instrumentos de cohesión e integración en época de crisis. ¿Utopía o realidad?
}

\author{
Mercedes Caridad Sebastián \\ Ana María Morales García \\ Fátima García López
}

Artículo recibido:

16 de agosto de 2013.

Artículo aceptado:

27 de febrero de 2014.

\section{Resumen}

Desde la década de 1990, las políticas europeas apostaron por la Sociedad de la Información y el Conocimiento como una de las acciones clave para potenciar el crecimiento, la competitividad y el empleo. En la actualidad, y bajo el nuevo escenario de incertidumbre económica y social, la Unión Europea, siguiendo la estrategia Europa 2020, continúa poniendo en marcha una serie de medidas para lograr una renovación en acciones prioritarias: Crecimiento inteligente (desarrollo de una economía basada en el conocimiento y la innovación), Crecimiento sostenible (promoción de una economía que haga un uso más eficaz de los recursos,

\footnotetext{
Las tres autoras pertenecen a la Universidad Carlos III de Madrid, España.

mercedes@bib.uc3m.es; amorales@bib.uc3m.es; fatimag@bib.uc3m.es 
que sea más ecológica y competitiva) y Crecimiento integrador (fomento de una economía con alto nivel de empleo que contemple la cohesión social y territorial). El éxito de la estrategia Europa 2020 dependerá de un enfoque integrador y coherente en todos los ámbitos de acción pertinentes, con especial atención a las políticas social, económica y de empleo.

Palabras clave: Europa 2020; Inclusión Social; Políticas de Información; Crecimiento inteligente, sostenible e integrador.

\section{Abstract}

Europe 2020 Strategy and the Information Society as tools of cohesion and integration in time of crisis: utopia or reality?

Mercedes Caridad-Sebastián, Ana-Maria Morales-García and Fátima García-López

Since the early 1990s, European policies pushed for the establishment of an Information and Knowledge Society as one of the key initiatives for growth, competitiveness and employment. Nowadays, under shadow new economic and social uncertainties, the European Union, pursuant to the Europe 2020 Strategy continues to push several priority measures, including: Smart growth (developing an economy based on knowledge and innovation), Sustainable growth (promoting a more resource efficient, greener and more competitive economy) and Inclusive growth (fostering a high-employment economy delivering social and territorial cohesion). The success of the Europe 2020 Strategy will depend on strong, cohesive policies, with special attention to social, economic and employment issues.

Keywords: Europe 2020; Social Inclusion; Information Polity; Smart, Sustainable and Inclusive Growth. 


\section{EL DESEMPLEO EN EUROPA: EVOLUCIÓN Y PERSPECTIVAS} ANTE LA SOCIEDAD DE LA INFORMACIÓN

T a Unión Europea, compuesta por seis Estados miembros en 1957, cuenta Len el año 2013 con 27 Estados, tras cinco ampliaciones. Si hacemos un análisis de los últimos años, podemos observar que ya en la década de 1990 la economía europea experimentaba una importante crisis estructural cuya repercusión más visible y preocupante fue el aumento de las tasas de desempleo en los distintos Estados miembros, que pasaron de un $2 \%$ en 1960 a un $12 \%$ en 1994, con las siguientes fluctuaciones en determinados periodos de crisis $^{1}$ (Gráfico 1):

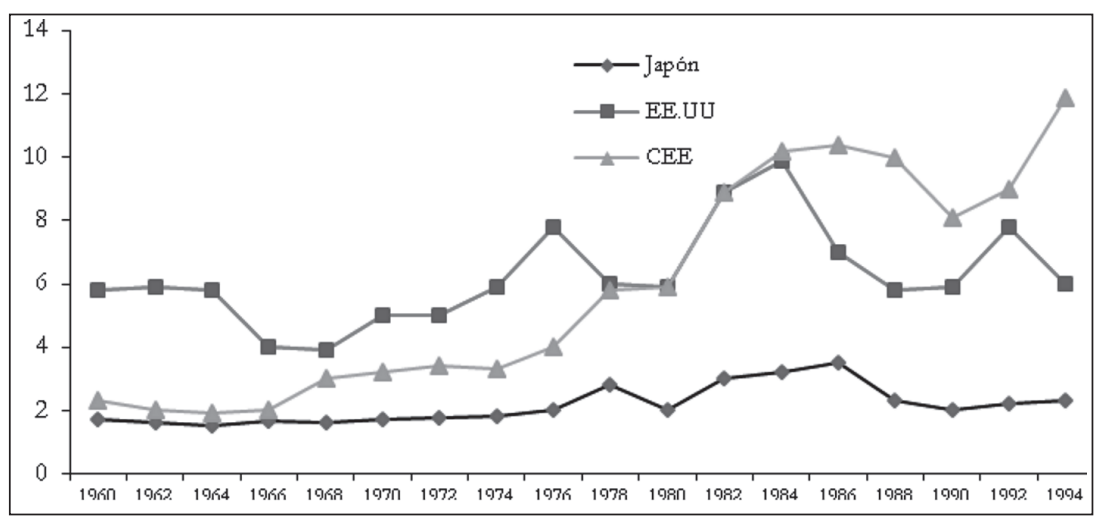

Gráfico 1. Comparativa del porcentaje de desempleo en Japón, EEUU y CEE Fuente: Comisión de las Comunidades Europeas, "Crecimiento, competitividad y empleo.

Retos y pistas para entrar en el siglo XXI. Libro Blanco", p. 41

- De 1960 a 1973 el aumento de las tasas de desempleo se mantuvo relativamente estable, por debajo de EEUU y muy cerca de Japón.

- En el periodo de 1974 a 1985 se produjo un importante incremento de la población activa. Disminuyó el empleo en el sector privado y aumentó en el sector público. Pese a ello la tasa de desempleo creció profundamente y alcanzó su nivel más elevado en el año 1985.

- Entre 1986 y 1990 se produjo una constante mejora, aunque las tasas no llegaron a los índices registrados en la década de 1960.

- A partir de 1991 comenzó a incrementarse el desempleo, que llegó en 1994 a un $12 \%$, el pico más alto registrado hasta ese momento.

1 Véase Comisión de las Comunidades Europeas, "Crecimiento, competitividad y empleo. Retos y pistas para entrar en el siglo XXI. Libro Blanco”. 
Ante esta situación, el Consejo encargó a la Unión Europea el estudio del problema y una propuesta de posibles soluciones, que dio como resultado en 1993 el conocido Libro Blanco de Delors Crecimiento, competitividad y empleo. Retos y pistas para entrar en el siglo XXI. El Libro Blanco de Delors (conocido así por la implicación personal en su materialización del Presidente de la Comisión, Jacques Delors) recibió el respaldo de la Cumbre Europea de Jefes de Estado y de Gobierno, celebrada en Bruselas los días 10 y 11 de diciembre de 1993.

El Libro Blanco dejó una clara constancia sobre la preocupación que existía respecto al desempleo y que, pese a lo arduo que resulta combatirlo, Europa deseaba responder con gran rapidez. Para ello, analizó la situación que había precedido a la década de 1990 y consideró a ésta última como una premisa importante para alcanzar la Sociedad de la Información, que con el apoyo incondicional del desarrollo de las "nuevas tecnologías" constituiría una acción clave para potenciar el crecimiento, la competitividad y el empleo.

Sin embargo, 20 años después, según datos estadísticos publicados por Eurostat, se puede concluir que el desempleo en Europa continúa hoy siendo un problema estructural. Comparando los datos estadísticos de desempleo con los de países como Japón y Estados Unidos, se observa que Europa continúa a la cabeza, con el mayor porcentaje de paro. Las tasas de desempleo en Japón $(4.6 \%$ ) y en Estados Unidos (8.1 \%) han sido más bajas que en la Unión Europea (11 \% en la EU-27 y $12.2 \%$ en la eurozona). Ésta ha sido la tendencia a lo largo de los últimos años, a excepción de 2009, cuando la tasa de desempleo en los Estados Unidos alcanzó el mismo nivel que en la Unión Europea (Gráfico 2).

Ante esta situación, es necesario que Europa aborde el tema del empleo y el crecimiento de manera más contundente. Tomando como referente el discurso pronunciado por el Presidente de la Comisión Europea, José Manuel Durão Barroso, en la presentación de la estrategia Europa 2020, ${ }^{2}$ se puede deducir que "la actual Europa 2020 será la estrategia de crecimiento de la Unión Europea para la próxima década. En un mundo en transformación, se pretende que la Unión Europea posea una economía inteligente, sostenible e integradora. Estas tres prioridades, que se refuerzan mutuamente, contribuirán a que la Unión Europea y sus Estados miembros generen altos niveles de empleo, productividad y cohesión social. Concretamente, la Unión ha establecido para 2020 cinco ambiciosos objetivos en materia de empleo, innovación, educación, integración social y clima/energía. En cada una de estas áreas, cada Estado miembro se ha fijado sus propios objetivos". Por otra parte, 


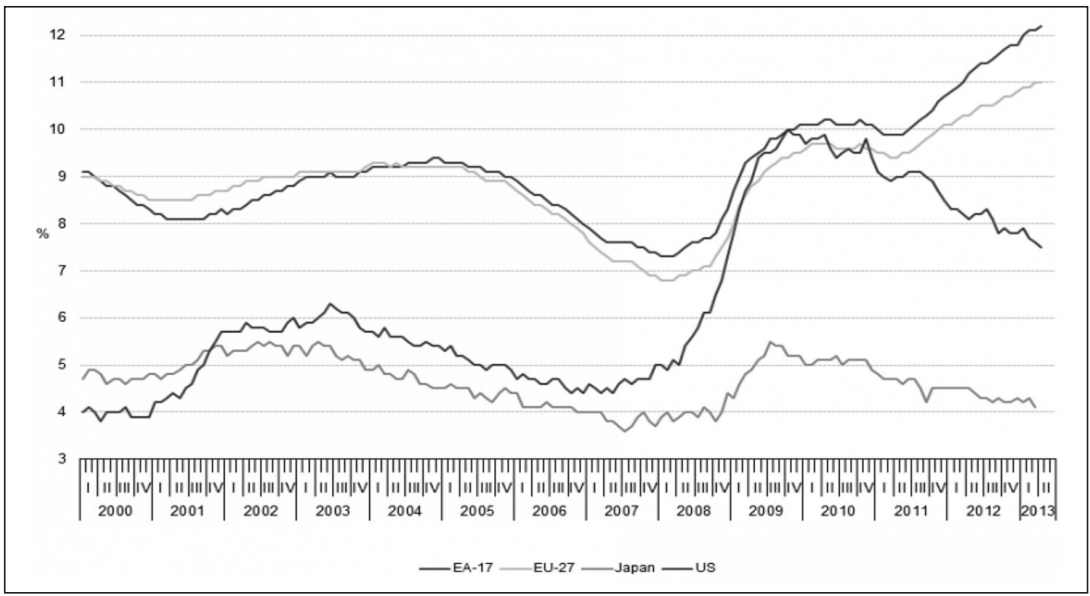

Gráfico 2. Las tasas de desempleo de la EA-17, EU-27, EEUU y Japón (2000-2013)

Fuente: Unemployment rates EU-27, EA-17, US and Japan, seasonally adjusted,

January 2000-April 2013 (abril 2013)

la Agenda Digital, como una de las primeras iniciativas emblemáticas de la estrategia Europa 2020, se centrará en las tecnologías y servicios en línea del siglo XXI que permitirán a Europa impulsar la creación de empleo, acelerar la recuperación económica, mejorar de diversas maneras la vida cotidiana de los ciudadanos y sentar las bases de un futuro digital sostenible.

Sin embargo, pese a los objetivos marcados en la estrategia comunitaria para el próximo periodo y hasta el año 2020, se abre una incógnita relacionada con el impacto que tendrán estas medidas en un futuro inmediato para paliar el desempleo y disminuir la brecha digital existente. En estos momentos, los países más afectados por la crisis económica y financiera (Irlanda, Portugal, Grecia, España e Italia) acentúan sus distancias respecto a los países de la Europa del Norte (Alemania, Holanda y países escandinavos). Se presentan tiempos convulsos y, si la situación económica de la Europa de los años 90 fue incierta e inquietante, la coyuntura que vivimos hoy se está convirtiendo en algo prácticamente alarmante.

\section{Los planes de acción de La Unión Europea COMO políticas DE INFORMACIÓN Y DE INCLUSIÓN SOCIAL}

Como hemos indicado, desde la década de 1990 las políticas europeas apostaron por la Sociedad de la Información y el Conocimiento como una de las acciones clave para potenciar el crecimiento, la competitividad y el empleo. 
Desde el año 2000 se vienen fortaleciendo una serie de acciones, programas e iniciativas comunitarias. Estas acciones se han fundamentado en planes de acción como eEurope (2002 y 2005), i2010-A European Information Society for growth and employment, Digital Agenda for Europe y Europe 2020 (Figura 1).

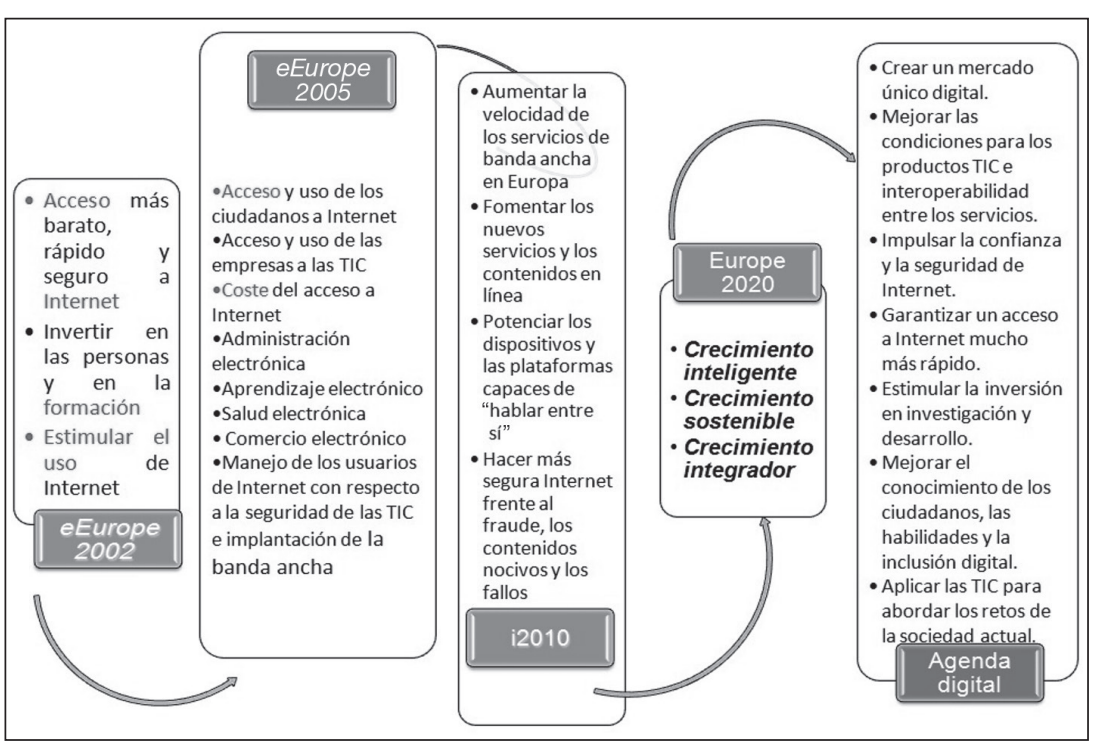

Figura 1. Objetivos de los planes de acción en Europa para el fomento de la Sociedad de la Información Fuente: Elaboración propia

eEurope-Una Sociedad de la Información para todos ha sido la iniciativa política dirigida a asegurar que la Unión Europea obtenga el máximo provecho de los cambios que está produciendo la Sociedad de la Información. El plan de acción eEurope 2002 se inscribió directamente en el marco de la estrategia de Lisboa, pensada para convertir a la Unión Europea en la economía del conocimiento más dinámica y competitiva del mundo. Las acciones se agruparon en torno a tres objetivos clave que debían alcanzarse para finales de 2002 (Figura 1). eEurope 2005, sucesor del plan eEurope 2002, fue aprobado por el Consejo Europeo de Sevilla en junio de 2002. Este plan pretendió traducir la conectividad a Internet en un aumento de la productividad económica y en una mejora de la calidad y la accesibilidad de los servicios, basándose en una infraestructura de banda ancha segura y disponible para la mayoría. Finalmente, MODINIS: seguimiento del plan de acción eEurope 2005 (2003-2006) cierra este periodo como programa plurianual que se propone la difusión de buenas prácticas, la comparación de los resultados entre los 
Estados miembros y el apoyo a las acciones de sensibilización destinadas a reforzar la seguridad de las redes y de la información.

i2010-A European Information Society for growth and employment (20052009), como plan de acción y marco estratégico de la Comisión Europea, determinó las orientaciones políticas generales de la Sociedad de la Información y los medios de comunicación. Propuso, en particular, fomentar el conocimiento y la innovación con el objetivo de lograr un crecimiento económico global y la creación de nuevos empleos. Su propósito fue coordinar la acción de los Estados miembros para facilitar la convergencia digital y afrontar los desafíos vinculados a la Sociedad de la Información. Para elaborar este marco estratégico, la Comisión llevó a cabo una amplia consulta con los agentes en torno a iniciativas e instrumentos anteriores, tales como eEurope.

Para el periodo 2010-2020, la Agenda Digital se enmarca en la estrategia Europa 2020 y constituye uno de sus siete pilares, que fija objetivos para el crecimiento de la Unión Europea hasta 2020. Europa necesita un nuevo plan de acción que suprima los obstáculos que actualmente impiden obtener un máximo rendimiento de las TIC y ahí debería estar la Agenda Digital, compuesta por siete líneas de acción, con sus correspondientes objetivos y acciones clave (Figura 1).

En junio de 2010, la Comisión Europea aprueba la estrategia Europa 2020 con el reto de sacar a la Unión Europea de la crisis y preparar su economía para la próxima década. Para ello ha identificado tres motores clave del crecimiento que deberán ponerse en marcha a través de acciones concretas en el ámbito nacional y de la Unión Europea: crecimiento inteligente, crecimiento sostenible y crecimiento integrador.

La iniciativa Europa 2020, como una estrategia para el empleo y el crecimiento inteligente, sostenible e integrador, basa sus cinco objetivos prioritarios (empleo, innovación, educación, integración social y clima/energía) en ocho indicadores principales (Tabla 1). En cada una de estas áreas cada Estado miembro se ha fijado sus propias metas.

Tabla 1. Indicadores Europa 2020

\begin{tabular}{|c|c|}
\hline Objetivos principales & Indicadores \\
\hline 1. Emplear al $75 \%$ de la población de 20-64. & Tasa de empleo por sexo (grupo de edad 20-64). \\
\hline $\begin{array}{l}\text { 2. Invertir el } 3 \text { \% del PIB de la UE en I+D (Investiga- } \\
\text { ción, Desarrollo e Innovación). }\end{array}$ & PIB en I+D. \\
\hline \multirow{3}{*}{$\begin{array}{l}\text { 3. Disminuir en un } 20 \% \text { las emisiones de gases de } \\
\text { efecto invernadero. } \\
\text { Aumentar la cuota de las energías renovables en un } \\
20 \% \text {. } \\
\text { Reducción del consumo de energía en un } 20 \% \text {. }\end{array}$} & $\begin{array}{l}\text { Emisiones de gases de efecto invernadero a los } \\
\text { niveles de } 1990 .\end{array}$ \\
\hline & $\begin{array}{l}\text { Participación de las energías renovables en el consu- } \\
\text { mo final bruto de energía. }\end{array}$ \\
\hline & Eficiencia energética. \\
\hline
\end{tabular}




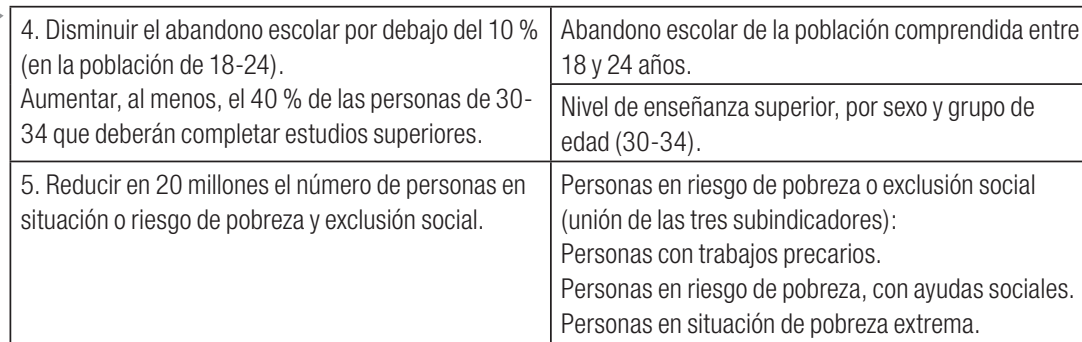

Fuente: Elaboración propia a partir de Comisión Europea, Europe 2020 indicators, Eurostat

La propia estrategia Europa 2020 incide en la interrelación de los objetivos propuestos:

Un mejor nivel educativo ayuda a encontrar trabajo y los avances en el aumento de la tasa de empleo ayudan a reducir la pobreza. Una mayor capacidad de investigación y desarrollo, así como la innovación en todos los sectores de la economía, combinada con una mayor eficacia de los recursos mejorarán la competitividad e impulsarán la creación de empleo. Invertir en tecnologías más limpias y con menores emisiones de carbono ayudará a nuestro medio ambiente, contribuirá a luchar contra el cambio climático y creará nuevas oportunidades empresariales y de empleo.

De los cinco objetivos marcados por Europa 2020, cuatro de ellos (Empleo, I+D, Educación y Lucha contra la pobreza y la exclusión social), directa o transversalmente, están vinculados al desarrollo de la Sociedad de la Información y pueden no ser alcanzados por la crisis económica, aspecto que incidirá negativamente en el avance hacia la Sociedad del Conocimiento.

a) Empleo

Se contempla que el nivel de empleo de la población entre 20 y 64 años aumente del $68.5 \%$ actual a por lo menos el $75 \%$ mediante, entre otros factores, una mayor participación de las mujeres y los trabajadores de mayor edad y una mejor integración de los inmigrantes en la población activa. Sin embargo, en el primer semestre de 2013, el desempleo ha alcanzado un nivel récord y la empleabilidad y la segmentación del mercado laboral constituyen "cuellos de botella" importantes. Se pronostica que el desempleo seguirá aumentando en los próximos años e incluirá a los jóvenes.

Un análisis detallado entre los Estados miembros nos muestra que las tasas de desempleo más bajas se registraron en Austria (4.9\%), Alemania $(5.4 \%)$ y Luxemburgo (5.6 \%), y las tasas más altas en Grecia (27 \%), España (26.8 \%) y Portugal (17.8 \%) (Gráfico 3). 


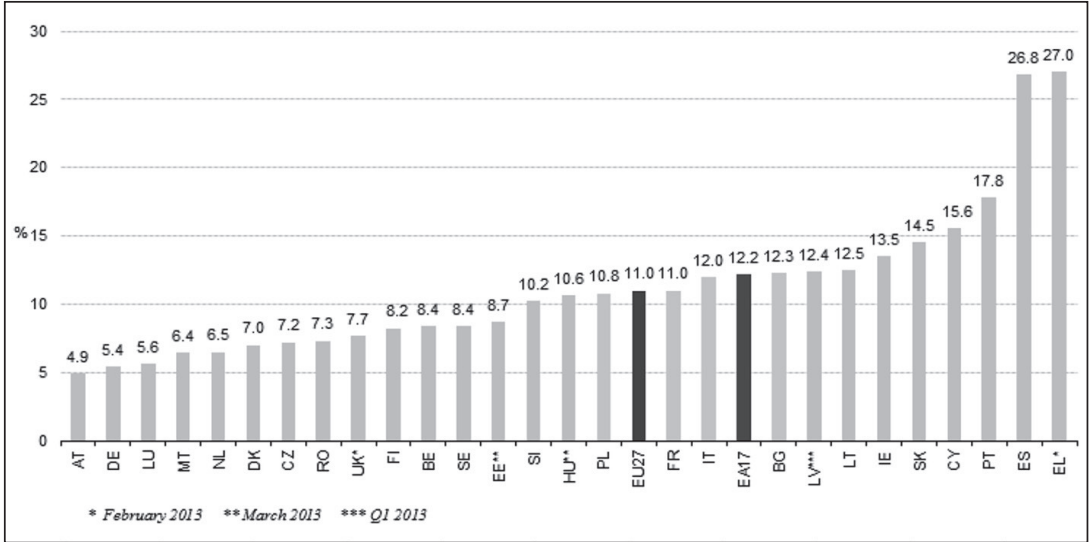

\section{Gráfico 3. Las tasas de desempleo en la Unión Europea Fuente: Unemployment rates seasonally adjusted-April 2013, Eurostat ${ }^{3}$}

\section{b) I+D +i (Investigación, Desarrollo e Innovación)}

El objetivo de la estrategia Europa 2020 incluye una inversión del $3 \%$ del PIB en I+D. Entre las medidas propuestas para alcanzar este objetivo, la Comisión Europea ha presentado el nuevo programa de I+D+i, Horizonte 2020 (2014-2020), ${ }^{4}$ dotado con 80 billones de euros. Horizonte 2020 proporcionará financiación en todas las etapas del proceso de innovación, desde la investigación básica hasta que el producto llegue al mercado. Se complementará con nuevas medidas que permitan definir el Espacio Europeo de Investigación en 2014 para crear un mercado único del conocimiento, la investigación y la innovación. La puesta en marcha de este nuevo programa de I+D+i sigue las directrices marcadas sobre el crecimiento inteligente, sostenible e integrador planteado en el actual Plan de Acción Europa 2020.

Según un estudio de la propia Unión, si se consigue para el año 2020 invertir el $3 \%$ del PIB de la Unión Europea en I+D, en el año 2025 se habrán creado 3.7 millones de empleos. Sin embargo, en estos momentos Europa se encuentra todavía a gran distancia de los objetivos marcados. La media de la Unión se sitúa en un discreto $2.03 \%$ y, únicamente, tres de los Estados miembros cumplen en la actualidad este objetivo: Finlandia (3.78 \%), Suecia (3.37 \%) y Dinamarca (3.09\%) (Gráfico 4).

3 Disponible en http://epp.eurostat.ec.europa.eu/statistics_explained/index.php/File:Unem ployment_rates,_seasonally_adjusted,_April_2013.png (Fecha de consulta: 15 de junio de 2013).

4 Véase Comisión Europea, Horizon 2020. 


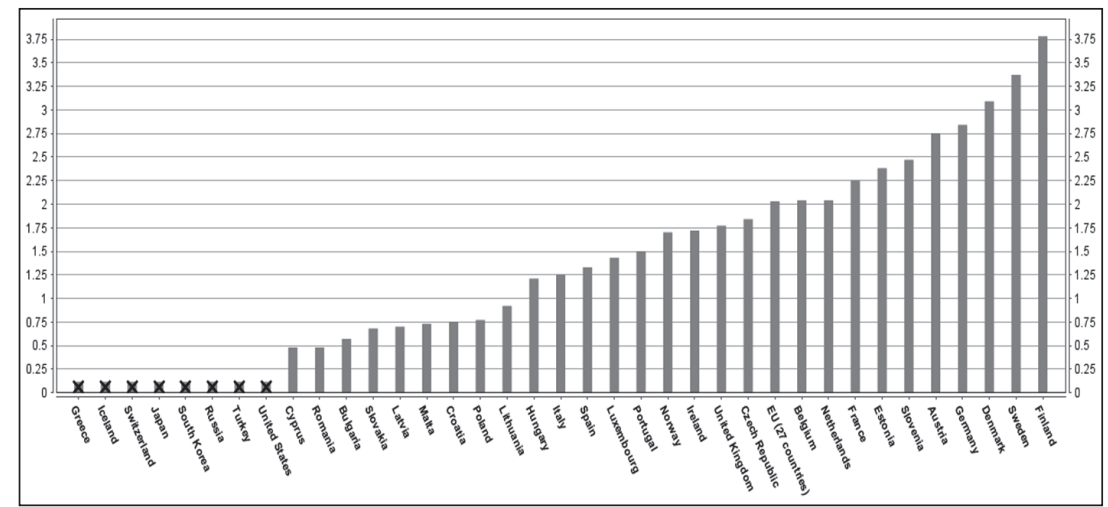

Gráfico 4. PIB en I+D
Fuente: Gross domestic expenditure on R\&D (GERD). Eurostat ${ }^{5}$

\section{c) Educación}

En lo que respecta al objetivo marcado por Europa 2020 para la Educación se contempla una disminución en las tasas de abandono escolar, por debajo de un $10 \%$, para la población comprendida entre 18 y 24 años, y el incremento en un $40 \%$ del porcentaje de población de entre 30 y 34 años con estudios superiores. Lograr estos resultados resulta hoy una cuestión de vital importancia puesto que repercuten directamente en la futura inserción laboral de los ciudadanos y en las políticas de empleo de los Estados miembros.

La reducción del fracaso escolar es una de las asignaturas pendientes del sistema educativo de varios países de la Unión Europea, donde destaca España con un $24.9 \%$ de tasa de abandono. A pesar de las acciones llevadas a cabo en los últimos años, España se encuentra a 12 puntos de la media europea (12.8\%), seguida por Malta (22.6\%), Portugal (20.8\%), Italia (17.6\%) y Rumanía (17.4\%). Es importante destacar que, actualmente, sólo 11 países cumplen con las tasas de abandono escolar por debajo de un $10 \%$. Este es el caso de Eslovenia, República Checa, Polonia, Lituania, Suecia, Austria, Luxemburgo, Holanda, Finlandia, Dinamarca e Irlanda (Gráfico 5). $\mathrm{de}=$ t2020_20\&language $=$ en $\&$ toolbox $=$ sort $($ Fecha de consulta: 15 de junio de 2013). 


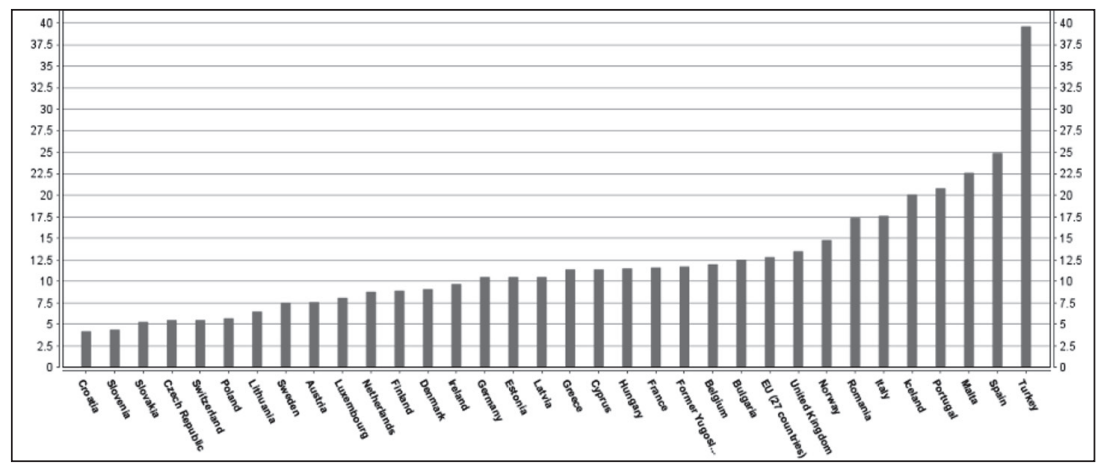

Gráfico 5. Porcentaje de población de 18 a 24 años con estudios secundarios

Fuente: Early leavers from education and training by sex. \% of the population aged 18-24 with at most lower secondary education and not in further education or training, Eurostat ${ }^{6}$

A diferencia del análisis anterior y por lo que respecta a los estudios superiores, es paradójico que la población con estudios universitarios en España se sitúa en un $40.1 \%$, porcentaje ligeramente superior al europeo $(35.8 \%)$ y también al alemán (31.9\%) (Gráfico 6). Sin embargo, el mercado laboral español no es capaz de generar tanto trabajo para ese nivel formativo, lo que supone un problema añadido a la situación actual de crisis económica que vive España, en la que muchos jóvenes con estudios superiores se ven obligados a emigrar para poder optar a un puesto de trabajo acorde con su nivel de formación.

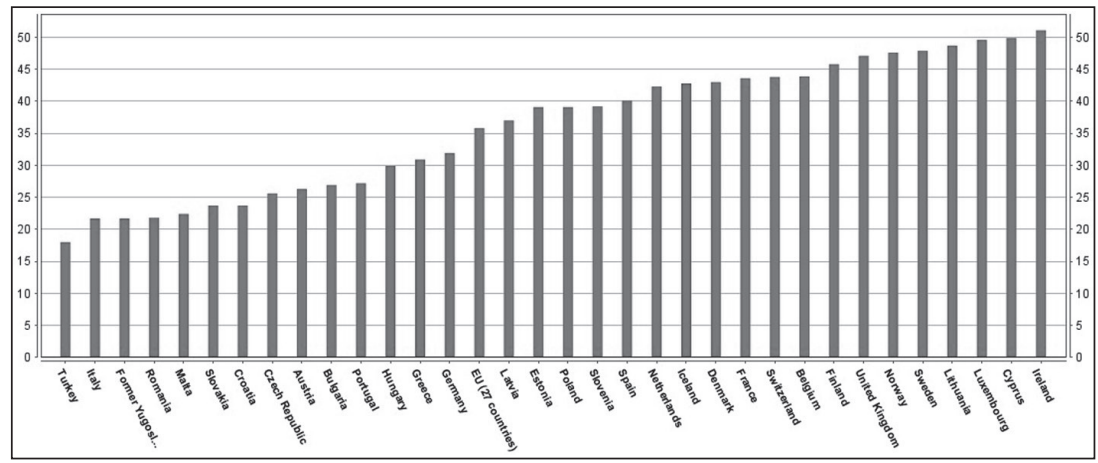

Gráfico 6. Porcentaje de población de 30 a 34 años con estudios superiores completos Fuente: Tertiary educational attainment. \% of population aged 30-34, Eurostat ${ }^{7}$

6 Disponible en: http://epp.eurostat.ec.europa.eu/tgm/graph.do?tab=graph\&plugin=0\&pco de $=$ t2020_40\&language $=$ en \&toolbox $=$ sort $($ Fecha de consulta: 15 de junio de 2013).

7 Disponible en: http://epp.eurostat.ec.europa.eu/tgm/graph.do?tab=graph\&plugin=0\&pco $\mathrm{de}=$ t2020_41\&language $=$ en \&toolbox $=$ sort $($ Fecha de consulta: 15 de junio de 2013). 
d) Lucha contra la pobreza y la inclusión social

Se pretende que el número de europeos que viven por debajo de los umbrales nacionales de pobreza se reduzca en un $25 \%$ y se rescate así a más de 20 millones de personas.

A través de las estadísticas que nos muestra Eurostat, se han podido determinar los valores relativos al número de personas en riesgo de pobreza o exclusión social, por países y desde diferentes puntos de vista (personas con trabajos precarios, personas en riesgo de pobreza con ayudas sociales y personas en situación de pobreza extrema). Por encima de la media europea $(24.2 \%)$ se observa un porcentaje muy alto en varios Estados de la Unión: Bulgaria (49.1\%), Letonia (40.4\%), Rumanía (40.3\%), seguidos muy de cerca por Lituania (33.4\%), Hungría (31\%), Grecia (31\%), Italia (28.2\%), Polonia (27.2 \%), España (27 \%) y Portugal (24\%) (Gráfico 7).

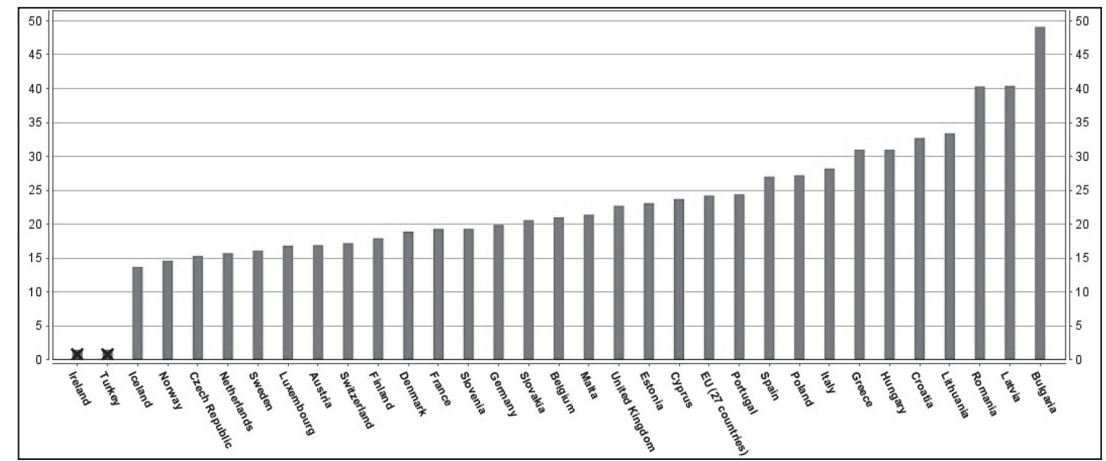

Gráfico 7. Personas en riesgo de pobreza o exclusión social Fuente: People at risk of poverty or social exclusion, Eurostat ${ }^{8}$

En el mismo sentido, el informe La dimensión social de la estrategia Europa $2020^{9}$ viene a subrayar la apuesta por superar los obstáculos de la economía europea; en concreto, alcanzar el objetivo de reducir el número de personas que se encuentran en situación de pobreza y riesgo de exclusión social. Este informe nos recuerda el "espeluznante" dato de que una de cada cinco personas en la Unión Europea están en riesgo de pobreza y exclusión social, aspecto que debilita la cohesión social y limita el potencial de los ciudadanos de Europa.

8 Disponible en: http://epp.eurostat.ec.europa.eu/tgm/graph.do?tab=graph\&plugin=0\&pco $\mathrm{de}=$ t2020_50\&language $=$ en \& toolbox $=$ sort $($ Fecha de consulta: 15 de junio de 2013).

9 La dimensión social de la estrategia Europa 2020, informe del Comité de Protección Social, ha recibido el apoyo del Programa de la Unión Europea para el Empleo y la Solidaridad Social, Progress (2007-2013). Disponible en: http://ec.europa.eu/progress 
Finalmente, el informe pone sobre la mesa que el éxito de la estrategia Europa 2020 dependerá de un enfoque integrador y coherente sobre todos los ámbitos de acción pertinentes, que ponga especial atención en las políticas social, económica y de empleo, y establezca una estrecha cooperación entre todos los niveles de gobierno, los interlocutores sociales y la sociedad civil. Si la ayuda financiera de la Unión Europea se vincula a las prioridades de Europa 2020 y respalda la innovación social, su efectividad será mayor.

Por lo que toca a España, podemos constatar que la alta tasa de desempleo (26.8 \% , según Eurostat) se ha convertido en la principal causa de pobreza y exclusión social, aspecto que en estos momentos parece imposible restaurar en su debilitada economía. El número de trabajadores sin recursos sigue aumentando y varios grupos de población están expuestos a un mayor riesgo de pobreza: padres de familias monoparentales que pierden su trabajo o que perciben salarios muy bajos, ancianos con bajos recursos, discapacitados, inmigrantes, mujeres, jóvenes y desempleados. Cada día aparecen nuevas y variadas formas de exclusión y llegamos a estadios que podríamos denominar "inquietantes y aterradores": exclusión en cuanto a la vivienda, la exclusión financiera y la precariedad en cuanto al acceso a la energía (electricidad y calefacción) y a los bienes domésticos de primera necesidad.

Afrontar todos estos retos pasa por una economía basada en el conocimiento y la innovación como impulsores de un "crecimiento inteligente". Para ello, tal como contempla la estrategia europea para los próximos años, es necesario mejorar la calidad de la educación, consolidar los resultados de la investigación, promover la innovación y la transferencia de conocimientos, explotar al máximo las TIC y asegurarse de que las ideas innovadoras puedan convertirse en nuevos productos y servicios que generen crecimiento y empleo de calidad en todos los Estados de la Unión.

\section{Conclusiones}

- Desde la década de 1990 las políticas europeas apostaron por la Sociedad de la Información y el Conocimiento como una de las acciones clave para potenciar el crecimiento, la competitividad y el empleo.

- Pese a los objetivos marcados en la estrategia Europa 2020, se abre una incógnita relacionada con el impacto que tendrán estas medidas, en un futuro inmediato, para paliar el desempleo y disminuir la brecha digital existente. En estos momentos, los países más afectados por la crisis económica y financiera (Irlanda, Portugal, Grecia, España e Italia) acentúan sus distancias respecto a los países de la Europa del Nor- 
te (Alemania, Holanda y países escandinavos). Ante esta situación es necesario que Europa aborde el tema del empleo y el crecimiento de manera más contundente.

- La estrategia europea para la próxima década aboga por una economía basada en el conocimiento y la innovación. En un mundo en transformación, se pretende que la Unión Europea posea una economía inteligente, sostenible e integradora. El éxito de la estrategia Europa 2020 dependerá de un enfoque integrador y coherente de todos los ámbitos de acción pertinentes, con especial atención a las políticas social, económica y de empleo. Sería deseable que lo planteado como estrategia para el año 2020 se materialice a través de acciones concretas y no sea una mera utopía.

\section{Bibliografía}

Caridad Sebastián, Mercedes, "Políticas de información para Educación: ámbito europeo”, en M. A. Marzal García-Quismondo y L. B. Andreu Lorenzo (coords.), La biblioteca: un mundo de recursos para el aprendizaje, Madrid, Ministerio de Educación y Ciencia, 2006, pp. 83-114.

__ Fátima García López y Ana María Morales García, "Europa ante la brecha digital", en I Conferencia Internacional Brecha Digital e Inclusión Social (27-29 de febrero de 2008, Instituto Universitario Agustín Millares y PROSIC).

__ y Ana María Morales García, "El papel de la sociedad de la información en el desarrollo científico", en Crítica, año 57, núm. 948, 2007, pp. 49-52.

Comisión de las Comunidades Europeas, "Crecimiento, competitividad y empleo. Retos y pistas para entrar en el siglo XXI. Libro Blanco", en Boletín de las Comunidades Europeas, Suplemento 6/93, Bruselas, Oficina de Publicaciones Oficiales de las Comunidades Europeas, 1993, 143 pp. Disponible en http://europa.eu/ documentation/official-docs/white-papers/pdf/growth_wp_ com_93_700_parts_a_b.pdf (Fecha de consulta: 15 de junio de 2013).

Comisión Europea, Comunicación de la Comisión. Europa 2020. Una estrategia para un crecimiento inteligente, sostenible e integrador, 2010. Disponible en: http://ec.europa.eu/commission_2010-2014 /president/news/documents/pdf/20100303_1_es.pdf (Fecha de consulta: 15 de junio de 2013).

__ Digital Agenda for Europe. Disponible en: http://ec.europa.eu/ information_society/digital-agenda/index_en.htm (Fecha de consulta: 15 de junio de 2013). 
—- Europa 2020. Disponible en: http://ec.europa.eu/europe2020/ index_es.htm (Fecha de consulta: 15 de junio de 2013).

— Europe 2020. Disponible en: http://ec.europa.eu/europe2020/ index_en.htm (Fecha de consulta: 15 de junio de 2013).

— Eurostat. Disponible en: http://epp.eurostat.ec.europa.eu/sta tistics_explained/index.php/Unemployment_statistics (Fecha de consulta: 15 de junio de 2013).

— - Eurostat. Europe 2020 indicators. Disponible en: http://epp.eu rostat.ec.europa.eu/portal/page/portal/europe_2020_indicators/headline_indicators (Fecha de consulta: 15 de junio de 2013).

— Eurostat. Unemployment statictics. Disponible en: http://epp. eurostat.ec.europa.eu/statistics_explained/index.php/Unemploy ment_statistics\#Recent_developments_in_unemployment_at_a_ European_and_Member_State_level (Fecha de consulta: 15 de junio de 2013).

— Horizon 2020. Disponible en: http://ec.europa.eu/research/ho rizon2020/index_en.cfm?pg=h2020 (Fecha de consulta: 15 de junio de 2013).

— , i2010-A European Information Society for growth and employment. Disponible en: http://ec.europa.eu/information_society/ eeurope/i2010/index_en.htm (Fecha de consulta: 15 de junio de 2013).

- Representación en España. I+D+i Investigación Desarrollo e Innovación. Disponible en: http://ec.europa.eu/spain/actualidad-yprensa/noticias/investigacion-desarrollo-e-innovacion/index_es. htm (Fecha de consulta: 15 de junio de 2013).

Morales García, Ana María, La Sociedad de la Información: políticas y acciones en Europa, Bogotá, Rojas Eberhard, 2008.

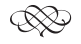

